\title{
Thermal solitons in the Toda chain
}

\author{
N. Theodorakopoulos and N. C. Bacalis \\ Theoretical and Physical Chemistry Institute, National Hellenic Research Foundation, Vas. Constantinou 48, \\ GR-116 35 Athens, Greece
}

(Received 30 June 1992)

\begin{abstract}
The fraction of degrees of freedom occupied by solitons in the classical Toda chain is evaluated as a function of temperature. Our results, derived within the framework of the Bethe ansatz (BA), are in complete agreement with recent numerical simulations that employ a soliton counter and reveal a $T^{1 / 3}$ dependence in the limit of low temperatures. The agreement confirms the existence of a $T$-independent soliton-phonon boundary in momentum space within the BA formalism, which apparently shares a common physical basis with the soliton counter's effective distinction between bound and scattering states.
\end{abstract}

The Toda chain has been an important model for testing fundamental aspects of nonlinearity; being a discrete lattice with the exact balancing of nonlinearity and dispersion, it can support pulselike soliton excitations. ${ }^{1}$ Because of its complete integrability property, ${ }^{2}$ it is amenable to an exact formulation of its statistical mechanics in terms of interacting solitons and phonons. ${ }^{3-7}$ Furthermore, it appears that some of its properties (e.g., soliton shape) represent generic features of large-amplitude motion in nonlinear lattices. ${ }^{8}$ As a consequence, results derived within the context of the Toda lattice can be reasonably expected to serve as useful guides for the behavior of real quasi-one-dimensional materials.

One of the first questions that can be asked about a soliton-bearing system at thermal equilibrium concerns the average number of thermally excited solitons. Muto, Scott, and Christiansen ${ }^{9}$ carried out a large-scale numerical simulation of Toda lattice dynamics and argued that, with a reasonable choice of parameters, their conclusions may have some relevance to the transport properties of DNA. Using a soliton counting technique, they determined the fraction of solitonlike degrees of freedom to be proportional to $T^{1 / 3}$ in the limit of low temperatures.

The $T^{1 / 3}$ power law has a rather long history. It was first derived in the context of the ideal soliton gas; ${ }^{10}$ the derivation was later shown to be erroneous ${ }^{11}$ (the correct $^{12}$ soliton density in this approximation is proportional to $T$ at low temperatures). It reappeared later within the framework of somewhat more sophisticated theories, which took into account the interaction of a single soliton with the thermal phonon gas. ${ }^{13-15}$ However, the predicted prefactors disagree with the results of the simulation and, what is more important, these one-soliton theories are known to fail in predicting other thermodynamic properties, e.g., specific heat or thermal expansion. Thus, once again, one cannot a priori exclude the possibility of a fortuitous result.

It is the purpose of this paper to use the Bethe-ansatz (BA) theoretical framework, which has been developed for the classical thermodynamics of the Toda lattice, ${ }^{3-7}$ in order to calculate an exact value for the soliton density as a function of temperature. Besides the obvious interest in comparing the results of the numerical simulation to those of an exact theory, we hope that this will deepen our understanding of dynamical processes at finite temperatures, by explicitly relating the microscopic dynamics predicted by the BA to the physical basis of the "soliton counter", 9 i.e., the fact that the soliton is a localized excitation.

In the following we shall give a concise account of the theoretical background on the statistical mechanics of the Toda chain, to the extent that is necessary in order to introduce concepts and notation used in this work. For details the reader is referred to the original literature. ${ }^{1-7}$

The Hamiltonian,

$$
H=\sum_{i=1}^{N}\left(\frac{p_{i}^{2}}{2 m}+\frac{a}{b} e^{-b\left(y_{i+1}-y_{i}\right)}+a\left(y_{i+1}-y_{i}\right)\right)
$$

describes a chain of $N$ atoms with equal mass $m$, coordinates $y_{i}$ and momenta $p_{i}$. The potential consists of a nearest-neighbor exponential repulsive interaction of range $b^{-1}$ and an attractive linear part, mathematically equivalent to an external force of strength $a$, which is responsible for holding the particles together in the lattice. In what follows, we shall use dimensionless quantities in which length is measured in units of $b^{-1}$, and energy in units of $a / b$; as a consequence, temperature is measured in units of $T_{0}=(a / b) / k_{B}$ and pressure in units of $a$; furthermore, we shall need the dimensionless coupling constant ${ }^{4}$ defined by the ratio of phonon-to-soliton energy, $g=\hbar \omega_{0} / k_{B} T_{0}$, where $\omega_{0}=\sqrt{a b / m}$.

The dynamical evolution of particle motion in the infinite Toda lattice can be described in terms of the inverse spectral transform (IST). ${ }^{2}$ In the context of the IST it is possible to identify two distinct types of dynamical behavior, according to the position of the eigenvalues in the spectrum of the operator associated with the particular nonlinear problem: (a) extended, subsonic, dispersive [phononlike] excitations arising from the continuum part, and (b) localized, supersonic, nondispersive [solitonlike] excitations arising from the discrete part of the spectrum of the associated linear operator. 
Scattering of any number of excitations is a completely elastic process and can be described by pairwise additive phase shifts. ${ }^{1,2}$ As a result, a complete description of equilibrium statistical mechanics can be given in terms of solitons, phonons, and their interactions, or, equivalently, in the framework of the BA. ${ }^{3-7}$ Thermodynamic properties can be expressed in terms of the distribution of momenta in $k$ space. The densities of occupied and unoccupied states are denoted by $\rho(k)$ and $\rho_{h}(k)$, respectively, and the ratio $\rho(k) / \rho_{h}(k) \equiv \exp (-\beta \epsilon(k))$, where $\beta$ is the (dimensionless) inverse temperature, defines a quasiparticle energy.

What is a thermal soliton within the BA framework? At zero temperature, particle states occupy all $k$ values up to a certain $k_{F}$. In the classical limit $\hbar \rightarrow 0$ it has been shown ${ }^{3}$ that $k_{F}=2 / g$ and the function $\rho(k)$ has a singularity at $k=k_{F}$; single-particle excitations correspond exactly to solitons or phonons, according to whether they are particlelike or holelike; the dispersion curves ${ }^{3}$ and other dynamical properties ${ }^{4-7}$ derived within the BA framework are identical with those of the IST.

At finite temperatures, a direct interpretation of the BA quasienergies is less clear. ${ }^{16,17}$ There is no singularity in either the $\rho(k)$ or the $\epsilon(k)$ curves. $^{4}$ In fact, it has been shown ${ }^{17}$ that in the space of the original quantum numbers defined in the BA context, the Fermi sea literally "evaporates" as soon as we heat the [classical] system. Nonetheless, it has been established ${ }^{5,7}$ that the classical limit of the BA description is exactly equivalent to the interacting soliton-phonon gas picture (i.e., a scheme in which solitons and phonons retain their identities as defined at $T=0$ ). The equivalence of these two distinct formulations of thermodynamics rests on the existence of a "Fermi momentum" $k_{F}$, which is defined at zero temperature ${ }^{7,18}$ and remains constant.

As a consequence, in spite of the absence of any singularity at $k_{F}$ within the BA formalism, an unambiguous procedure for counting thermal solitons is suggested: to count all states with $|k|>k_{F}$ as solitons. If such a procedure yields the same results with the soliton counter of Ref. 9 at all temperatures, this would imply that $k_{F}$ retains some physical significance even within the context of an "evaporated" Fermi sea.

Given a chain of $N$ atoms and length $L$, the number of solitons $N_{s}$ is defined (cf. above) via

$$
\frac{N_{s}}{L}=\int_{|k|>k_{F}} d k \rho(k) \text {. }
$$

In order to determine the soliton fraction $n_{s}=N_{s} / N$ we shall further use: (i) the relationship ${ }^{19}$

$$
\rho_{h}(k)=-\frac{1}{2 \pi} d\left[\frac{\partial \epsilon}{\partial p}\right]_{T}
$$

valid in the classical limit, where $d=N / L$ is the density and, (ii) the closed-form solution ${ }^{6}$ of the integral equation, ${ }^{4}$ which determines the quasiparticle energy $\epsilon(k)$ under the condition of given external pressure $p$, i.e., $e^{-\beta \epsilon(k ; \beta, p)}=2 g \beta^{1 / 2}\left[\frac{\partial}{\partial t} \theta[t, \beta(1+p)]\right]_{t=2 \beta^{1 / 2} k / k_{F}}$,

where

$$
\theta(t, \lambda)=\arg f_{+}(t, \lambda)
$$

and

$$
f_{+}(t, \lambda) \equiv f_{1}+i f_{2}=\int_{0}^{\infty} d u e^{i t u-u^{2} / 2} u^{\lambda-1}
$$

is a solution of

$$
\frac{d^{2} f_{+}}{d t^{2}}+t \frac{d f_{+}}{d t}+\lambda f_{+}=0
$$

(parabolic cylinder function). Using Eqs. (2)-(5) and Eqs. (12) and (28) of Ref. 6, it is straightforward to establish that

$$
\begin{aligned}
n_{s} & =\frac{2}{\pi} \int_{2 \sqrt{\beta}}^{\infty} d t\left[\frac{\partial^{2} \theta(t, \lambda)}{\partial \lambda \partial t}\right]_{\lambda=\beta(1+p)} \\
& =1-\frac{2}{\pi}\left[\frac{\partial \theta(2 \sqrt{\beta}, \lambda)}{\partial \lambda}\right]_{\lambda=\beta(1+p)} .
\end{aligned}
$$

The expression (8) represents the prediction of the exact (BA) statistical mechanics for the fraction of degrees of freedom occupied by solitons. Using the known ${ }^{20}$ asymptotic expansions for $f_{1}$ and $f_{2}$, we have derived the leading-order behavior of (8) for $p=0$ and $T \rightarrow 0$ :

$$
n_{s} \sim A T^{1 / 3}+B T^{2 / 3}+O(T),
$$

where $A=2 \times 3^{1 / 3} /[\Gamma(1 / 3)]^{2}=0.4019$. The simplest way to extract from the theory accurate results valid at all temperatures is via numerical integration of the nonlinear (Ricatti) equation, which arises in the context of the closed-form solution ${ }^{6}$ and turns out to have a much smoother behavior than the integral on the right-hand side of Eq. (6). If $f_{+}=F e^{i \theta}$, and $w=F^{\prime} / F$, then (7) is equivalent to

$$
\begin{aligned}
& \frac{d \theta}{d t} \equiv \psi, \\
& \frac{d \psi}{d t}=-(t+2 w) \psi, \\
& \frac{d w}{d t}=-w(w+t)+\psi^{2}-\lambda,
\end{aligned}
$$

with initial conditions

$$
\begin{aligned}
& \theta(0)=0, \\
& \psi(0)=f_{2}^{\prime}(0) / f_{1}(0)=\sqrt{2} \frac{\Gamma[(\lambda+1) / 2]}{\Gamma(\lambda / 2)}, \\
& w(0)=0 .
\end{aligned}
$$

The initial value problem defined by Eqs. (10)-(15) was solved numerically with a high degree of accuracy using the Bulirsch-Stoer method ${ }^{21}$ and for a number of adjacent values of $\lambda$. The fraction of degrees of freedom occupied by phonons under conditions of vanishing external pressure $p$ is given by the last term in Eq. (8) [computed with 


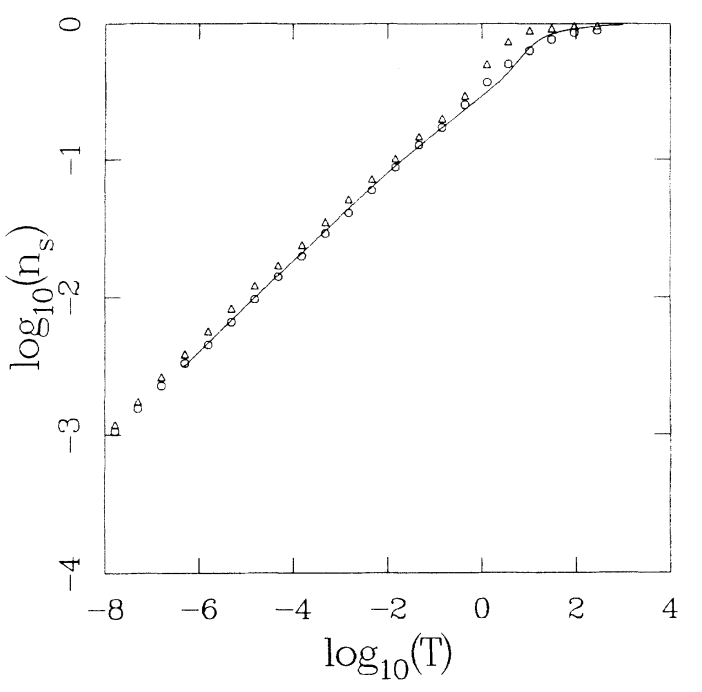

FIG. 1. The fraction of degrees of freedom $n_{s}$ occupied by solitons as a function of temperature plotted on logarithmic scales. Also plotted are results extracted from Fig. 1 (circles) and Fig. 2 (triangles) of Ref. 9(b), appropriately rescaled to our dimensionless units; the exact relationship (cf. Ref. 1) between energy (the nominal temperature of Ref. 9) and temperature has been taken into account. We attribute the residual discrepancies (more pronounced at the high-temperature regime) to the fact that constant energy simulation runs do not exactly reflect the behavior of a constant temperature system.

a relative accuracy of $\left.O\left(10^{-8}\right)\right]$. The rest is solitons. The results are plotted in Fig. 1 together with two (appropriately scaled) curves from Ref. 9. The agreement with the simulations is satisfactory over the entire temperature range. An analysis of the low-temperature results (Fig. 2) confirms the $T^{1 / 3}$ law and provides a value for the prefactor $A=0.402$ in excellent agreement with the result of the asymptotic expansion (9), as well as an estimate $B=-0.148$ for the leading correction term.

We have presented a theoretical calculation of the density of thermal solitons in the Toda lattice, based on the classical limit of the thermodynamics of the Betheansatz, and the concept of a temperature-independent boundary in momentum space, separating phononlike

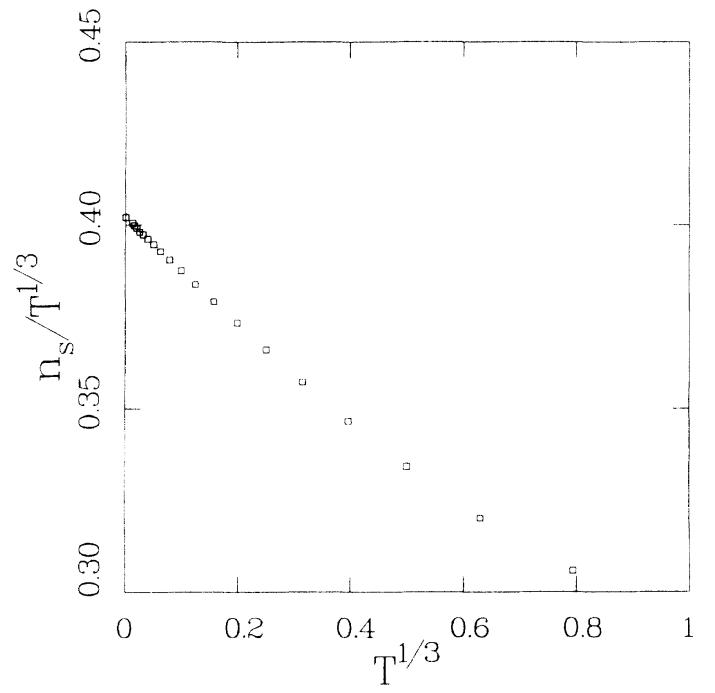

FIG. 2. The ratio $n_{s} / T^{1 / 3}$ plotted as a function of $T^{1 / 3}$. The results extrapolate to a value $A=0.402$ (Richardson extrapolation $^{21}$ ) in the limit $T \rightarrow 0$. The limiting slope provides a value for the coefficient $B=-0.148$ in the asymptotic expansion (9).

from solitonlike excitations. The agreement of our results with the numerical simulations suggests that our procedure shares a common physical basis with the classical soliton counter, ${ }^{9}$ which in effect tests whether a particular excitation is extended or localized.

Our [exact] results confirm that in the case of the Toda chain - a key model of lattice anharmonicity -, the most elementary global manifestation of nonlinearity, i.e., the extent to which nonlinear excitations occupy available phase space at finite temperatures, occurs in the form of a simple power law. In this context, the fact that the $1 / 3$ exponent can be derived by approximate theories, ${ }^{13-15}$ which do not invoke exact integrability, but are based on more generic features (e.g., phase shifts) of a single [nontopological] soliton's interaction with thermal phonons, prompts the speculative question: is there a kind of universality in the statistical properties of nonlinear lattices?
${ }^{1}$ M. Toda, Theory of Nonlinear Lattices (Springer, Berlin, 1981), and references cited therein.

${ }^{2}$ H. Flashka, Phys. Rev. B 9, 1924 (1974).

${ }^{3}$ B. Sutherland, Rocky Mount. J. Math. 8, 413 (1978).

${ }^{4}$ N. Theodorakopoulos, Phys. Rev. Lett. 53, 874 (1984).

${ }^{5}$ N. Theodorakopoulos, in Dynamical Problems in Soliton Systems, edited by S. Takeno (Springer, Berlin, 1985), p. 115.

${ }^{6}$ M. Opper, Phys. Lett. A 112, 201 (1985).

${ }^{7}$ H. Takayama and M. Ishikawa, Prog. Theor. Phys. 76, 820 (1986).

${ }^{8}$ T. J. Rolfe, S. A. Stuart, and J. Dancz, J. Chem. Phys. 70, 26 (1979).

${ }^{9}$ (a) V. Muto, A. C. Scott, and P. L. Christiansen, Phys. Lett. A
136, 33 (1989); (b) Physica D 44, 75 (1990).

${ }^{10}$ T. Schneider and E. Stoll, Phys. Rev. Lett. 45, 997 (1980); (unpublished).

${ }^{11} \mathrm{H}$. Bolterauer and M. Opper, Z. Phys. B 42, 155 (1981).

${ }^{12}$ F. G. Mertens and H. Büttner, Phys. Lett. A 84, 335 (1981).

${ }^{13}$ F. Yoshida and T. Sakuma, Phys. Rev. A 25, 2750 (1982).

${ }^{14} \mathrm{~N}$. Theodorakopoulos, in Statics and Dynamics of Nonlinear Systems, edited by H. Bilz et al. (Springer, Berlin, 1983), p. 271.

${ }^{15}$ F. Marchesoni and C. Lucheroni (ML) [Phys. Rev. B 44, 5303 (1991)], present a calculation of the soliton density based on the same physical input as Refs. 13 and 14. The ML key result, i.e. Eq. (10), was derived in Ref. 14 (Eq. 23) and appears 
in Ref. 4 [thermal renormalization of the soliton energy due to phonons]. However, the soliton density given by Eq. (13) of $\mathrm{ML}$ and corresponding to a prefactor $A=\Gamma(4 / 3)(3 / 4)^{1 / 3} / \pi=.258$ (note the different definitions of $T_{0}$ used by $\mathrm{ML}$ and in this work) misses a factor of 2 . As a consequence, the proposed "Fermi-liquid" correction (due to effects of the soliton-soliton interaction) by an overall factor of $e^{1 / 3}$, while apparently improving the [erroneously underestimated] result of Eq. (13) of ML to $A=0.360$, actually corresponds to a value of $A=0.720$ and hence to even larger deviations from the values of Ref. 9 (and/or the exact values reported here). The effects of soliton-soliton interactions [including the kinematical part that ensures compliance with important sum rules (cf. Ref. 14)] are, in fact, quite complicated to treat separately, even in an approximate manner.

${ }^{16}$ P. Grüner-Bauer and F. G. Mertens, Z. Phys. B 70, 435 (1988).
${ }^{17}$ M. Fowler and N-C. Yu, J. Phys. A 22, 3095 (1989).

${ }^{18} \mathrm{~N}$. Theodorakopoulos, in Proceedings of the 2nd International Conference on Phonon Physics, edited by J. Kollar et al. (World Scientific, Singapore, 1985), p. 468.

${ }^{19}$ C. N. Yang and C. P. Yang, J. Math. Phys. 10, 1115 (1969). Our Eq. (4) is a direct consequence of Eq. (24) of Yang and Yang, of the fact ${ }^{4}$ that in the classical limit $\rho(k) / \rho_{h}(k) \rightarrow 0$, and of the thermodynamic identity $d=(\partial p / \partial \mu)_{T}$ relating density, pressure, temperature, and chemical potential $\mu$.

${ }^{20}$ Handbook of Mathematical Functions, edited by $\mathbf{M}$. Abramowitz and I. A. Stegun (Dover, New York, 1965), p. 509.

${ }^{21}$ W. H. Press et al., Numerical Recipes (Cambridge University, Cambridge, England, 1986). In order to facilitate the computation for large values of $\lambda$, we used rescaled independent and dependent variables, $t=\sqrt{\lambda} \widetilde{t}, w=\sqrt{\lambda} \widetilde{w}, \psi=\sqrt{\lambda} \tilde{\psi}$, and $\theta=\lambda \widetilde{\theta}$. 